\title{
OGLE 2008-BLG-290: an accurate measurement of the limb darkening of a galactic bulge K Giant spatially resolved by microlensing
}

\author{
P. Fouqué ${ }^{1,44}$, D. Heyrovský ${ }^{48}$, S. Dong ${ }^{2,7}$, A. Gould ${ }^{2,7}$, A. Udalski ${ }^{3,27}$, M. D. Albrow ${ }^{1,8}$, V. Batista ${ }^{1,11}$, \\ J.-P. Beaulieu ${ }^{1,11}$, D. P. Bennett ${ }^{1,4,15}$, I. A. Bond ${ }^{4,30}$, D. M. Bramich ${ }^{1,5,10}$, S. Calchi Novati ${ }^{50}$, A. Cassan ${ }^{1,12}$, \\ C. Coutures ${ }^{1,11}$, S. Dieters ${ }^{1,11}$, M. Dominik ${ }^{1,5,6,13, \star}$, D. Dominis Prester ${ }^{1,17}$, J. Greenhill ${ }^{1,19}$, K. Horne ${ }^{1,5,13}$,
} U. G. Jørgensen ${ }^{6,45}$, S. Kozłowski ${ }^{2,7}$, D. Kubas ${ }^{1,9}$, C.-H. Lee ${ }^{46,61}$, J.-B. Marquette ${ }^{1,11}$, M. Mathiasen ${ }^{6,45}$, J. Menzies ${ }^{1,22}$, L. A. G. Monard ${ }^{2,26}$, S. Nishiyama ${ }^{59}$, I. Papadakis ${ }^{2,31}$, R. Street ${ }^{1,5,39,43}$, T. Sumi ${ }^{4,31}$, A. Williams ${ }^{1,21}$, J. C. Yee ${ }^{2,7}$, S. Brillant ${ }^{9}$, J. A. R. Caldwell ${ }^{15}$, A. Cole ${ }^{19}$, K. H. Cook ${ }^{16}$, J. Donatowicz ${ }^{18}$, N. Kains ${ }^{5,13}$, S. R. Kane ${ }^{20}$, R. Martin ${ }^{21}$, K. R. Pollard ${ }^{8}$, K. C. Sahu ${ }^{23}$, Y. Tsapras ${ }^{5,39,60}$, J. Wambsganss ${ }^{6,12}$, M. Zub ${ }^{6,12}$ (The PLANET Collaboration) ${ }^{1}$, D. L. DePoy ${ }^{7}$, B. S. Gaudi ${ }^{7}$, C. Hann $^{24}$, C.-U. Lee ${ }^{25}$, B.-G. Park ${ }^{25}$, R. W. Pogge ${ }^{7}$ (The $\mu$ FUN Collaboration) ${ }^{2}$, M. Kubiak ${ }^{27}$, M. K. Szymański ${ }^{27}$, G. Pietrzyński ${ }^{27,28}$, I. Soszyński ${ }^{27}$, O. Szewczyk ${ }^{27,28}$, K. Ulaczyk ${ }^{27}$, Ł. Wyrzykowski ${ }^{29}$ (The OGLE Collaboration) $^{3}$, F. Abe ${ }^{31}$, A. Fukui ${ }^{31}$, K. Furusawa ${ }^{31}$, A. C. Gilmore ${ }^{8}$, J. B. Hearnshaw ${ }^{8}$, Y. Itow ${ }^{31}$, K. Kamiya ${ }^{31}$, P. M. Kilmartin ${ }^{29}$, A. V. Korpela ${ }^{38}$, W. Lin ${ }^{8}$, C. H. Ling ${ }^{18}$, K. Masuda ${ }^{31}$, Y. Matsubara ${ }^{31}$, N. Miyake ${ }^{31}$, Y. Muraki ${ }^{33}$, M. Nagaya ${ }^{31}$, K. Ohnishi ${ }^{34}$, T. Okumura ${ }^{31}$, Y. Perrott ${ }^{35}$, N. J. Rattenbury ${ }^{5,35}$, To. Saito ${ }^{36}$, T. Sako ${ }^{31}$, S. Sato ${ }^{37}$, L. Skuljan ${ }^{8}$, D. Sullivan ${ }^{38}$, W. Sweatman ${ }^{8}$, P. J. Tristram ${ }^{29}$, P. C. M. Yock ${ }^{35}$ (The MOA Collaboration) ${ }^{4}$, A. Allan ${ }^{41}$, M. F. Bode ${ }^{40}$, M. J. Burgdorf $6,57,58$, N. Clay ${ }^{40}$, S. N. Fraser ${ }^{40}$, E. Hawkins ${ }^{39}$, E. Kerins ${ }^{32}$, T. A. Lister ${ }^{39}$, C. J. Mottram ${ }^{40}$, E. S. Saunders ${ }^{39,41}$, C. Snodgrass ${ }^{6,9}$, I. A. Steele ${ }^{40}$, P. J. Wheatley ${ }^{42}$ (The RoboNet-II Collaboration) $^{5}$, T. Anguita ${ }^{12}$, V. Bozza ${ }^{50},{\text { K. Harps } ø e^{45} \text {, T. C. Hinse }}^{45,51}$,

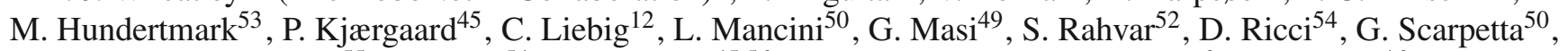
J. Southworth ${ }^{55}$, J. Surdej ${ }^{54}$, C. C. Thöne ${ }^{45,56}$ (The MiNDSTEp Consortium) ${ }^{6}$, A. Riffeser ${ }^{46}$, S. Seitz ${ }^{46,47}$, and R. Bender ${ }^{46,47}$ (The WeCAPP collaboration) ${ }^{61}$

(Affiliations can be found after the references)

Received 13 January 2010 / Accepted 5 May 2010

\section{ABSTRACT}

Context. Not only is gravitational microlensing a successful tool for discovering distant exoplanets, but it also enables characterization of the lens and source stars involved in the lensing event.

Aims. In high-magnification events, the lens caustic may cross over the source disk, which allows determination of the angular size of the source and measurement of its limb darkening.

Methods. When such extended-source effects appear close to maximum magnification, the resulting light curve differs from the characteristic Paczyński point-source curve. The exact shape of the light curve close to the peak depends on the limb darkening of the source. Dense photometric coverage permits measurement of the respective limb-darkening coefficients.

Results. In the case of the microlensing event OGLE 2008-BLG-290, the K giant source star reached a peak magnification at about 100. Thirteen different telescopes have covered this event in eight different photometric bands. Subsequent light-curve analysis yielded measurements of linear limb-darkening coefficients of the source in six photometric bands. The best-measured coefficients lead to an estimate of the source effective temperature of about $4700_{-200}^{+100} \mathrm{~K}$. However, the photometric estimate from colour-magnitude diagrams favours a cooler temperature of $4200 \pm 100 \mathrm{~K}$. Conclusions. Because the limb-darkening measurements, at least in the CTIO/SMARTS2 $V_{\mathrm{s}^{-}}$and $I_{\mathrm{s}}$-bands, are among the most accurate obtained, the above disagreement needs to be understood. A solution is proposed, which may apply to previous events where such a discrepancy also appeared.

Key words. gravitational lensing: micro - techniques: high angular resolution - stars: atmospheres - stars: individual: OGLE 2008-BLG-290

\section{Introduction}

Astrophysical opportunities for measuring stellar limb darkening are very scarce. Moreover, they are often limited to very specific types of stars. Until recently, most measurements had been based on analyses of eclipsing-binary light curves

^ Royal Society University Research Fellow.
(Popper 1984), requiring large amounts of high-precision photometric data but only rarely yielding useful constraints on the recovered limb-darkening parameters (e.g. Popper \& Etzel 1981; Southworth et al. 2005).

Resolving stellar surfaces by interferometry is a promising technique, limited nevertheless at present to nearby giants and supergiants. For most of the sufficiently well-resolved stars 
it has been shown that a fixed model-atmosphere-based limbdarkening profile agrees with the observations better than a uniform or fully limb-darkened stellar disk. Only a limited number of cases have been used to actually measure limb darkening from interferometric data (e.g., Burns et al. 1997; Perrin et al. 2004; Aufdenberg et al. 2006; Wittkowski et al. 2006). The same is true for observations of lunar occultation of stars, which only rarely yield limb-darkening measurements (Richichi \& Lisi 1990).

An alternative method that permits accurate limb-darkening measurement practically independent of stellar type is based on gravitational microlensing. In a stellar gravitational microlensing event the flux from an observed source star is temporarily amplified by the gravitational field of another object passing in the foreground and acting as a gravitational lens (Paczyński 1996). The lensing object may be a single star, a binary star, or a star with one or several planetary companions. A particularly interesting situation occurs in so-called caustic-crossing events, in which the caustic of the lens directly crosses the projected disk of the source star, typically on a timescale of a fraction of a day to a few days. The very high angular resolution provided by the caustic presents a unique opportunity to measure the source star's limb darkening from photometric observations or the centre-to-limb variation of spectral features from spectroscopic observations of the crossing (e.g. Witt 1995; Heyrovský et al. 2000; Heyrovský 2003).

The first well-documented source-resolving, causticcrossing microlensing event was MACHO Alert 95-30 (Alcock et al. 1997), in which an M4 III giant source star was scanned by the point-like caustic of a single lens. The observations permitted measurement of the source size and detection of its limb darkening. Precise measurement of the limb darkening was not possible due to low-amplitude variability of the source (Heyrovský 2003). The first limb-darkening measurement came from the analysis of binary-lens event MACHO 97-BLG-28 (Albrow et al. 1999), producing $V$ - and $I$-band coefficients of the linear and square-root limb-darkening laws for the K2 III giant source. By the time of writing, six further binary-lens caustic-crossing events yielded limb-darkening measurements of their source stars: MACHO 97-BLG-41 (Albrow et al. 2000), MACHO 98-SMC-1 (Afonso et al. 2000), OGLE 1999-BUL-23 (Albrow et al. 2001), EROS 2000-BLG-5 (An et al. 2002; Fields et al. 2003), MOA 2002-BLG-33 (Abe et al. 2003), and OGLE 2002-BLG-069 (Cassan et al. 2004; Kubas et al. 2005).

Limb-darkening measurements are also available from four single-lens, caustic-crossing events: OGLE 2003-BLG-262 (Yoo et al. 2004), OGLE 2003-BLG-238 (Jiang et al. 2004), OGLE 2004-BLG-254 (Cassan et al. 2006; Heyrovský 2008), and OGLE 2004-BLG-482 (Zub et al. 2009). In addition, there are a comparable number of events that required including limb darkening in their analysis (such as MACHO Alert 95-30), but for which a sufficiently accurate limb-darkening measurement was not (and often could not be) performed. These include two other single-lens events (Yee et al. 2009; Batista et al. 2009), as well as all ten published planetary microlensing events, in which a star with a planet acted as the lens (Bond et al. 2004; Udalski et al. 2005; Beaulieu et al. 2006; Gould et al. 2006; Gaudi et al. 2008; Bennett et al. 2008; Dong et al. 2009; Sumi et al. 2010; Janczak et al. 2010).

In this paper we report the results of the analysis of OGLE 2008-BLG-290, a microlensing event in which the caustic of a single lens crossed the disk of a $\mathrm{K}$ giant in the galactic bulge. In Sect. 2 we describe the obtained data and their reduction. We discuss the properties of the source star in detail in Sect. 3. The data modelling in Sect. 4 is followed in Sect. 5 by a description of the limb-darkening measurements in six photometric bands and their comparison with model atmospheres. We devote Sect. 6 to the study of the properties of the lens. We explore the possibility of deviations caused by a potential planetary companion to the lens in Sect. 7 and consider other potential systematics in Sect. 8. The main results are summarized in Sect. 9.

\section{Data sets: observations and data reductions}

The OGLE-III early warning system (EWS) (Udalski 2003) alerted the Bulge event OGLE 2008-BLG-290 $(\alpha=$ $17^{\mathrm{h}} 58^{\mathrm{m}} 29.3^{\mathrm{s}}, \delta=-27^{\circ} 59^{\prime} 22^{\prime \prime}$. (J2000.0) and $l=2.256^{\circ}, b=$ $-1.951^{\circ}$ ) on May 15,2008 , from observations carried out with the $1.3 \mathrm{~m}$ Warsaw Telescope at the Las Campanas Observatory (Chile). Independently, the MOA-II $1.8 \mathrm{~m}$ telescope at Mount John Observatory (New Zealand) discovered the same event under name MOA 2008-BLG-241 on May 31, 2008.

A few days later, it was clear that this event had the potential to become one of the rare high-magnification events discovered each year, and follow-up observations were undertaken on PLANET, $\mu$ FUN, RoboNet/LCOGT and MiNDSTEp telescopes available at that time. In total, 13 telescopes covered the event in different photometric bands: OGLE $1.3 \mathrm{~m}$ ( $I$-band), MOA-II $1.8 \mathrm{~m}$ (wide MOA-red band), SAAO $1.0 \mathrm{~m}$ at Sutherland (South Africa) ( $V$ - and $I$-bands), Canopus $1.0 \mathrm{~m}$ at Hobart (Australia) (I-band), Perth/Lowell $0.6 \mathrm{~m}$ at Bickley (Australia) (I-band), CTIO $1.3 \mathrm{~m}$ at Cerro Tololo (Chile) ( $V_{\mathrm{s}^{-}}, I_{\mathrm{s}^{-}}$and $H$-bands), LOAO $1.0 \mathrm{~m}$ at Mount Lemmon (Arizona) (I-band), Bronberg $0.36 \mathrm{~m}$ at Pretoria (South Africa) (unfiltered), Skinakas $1.3 \mathrm{~m}$ at Mount Ida (Greece) (I-band), Faulkes North $2.0 \mathrm{~m}$ at Haleakala (Hawaii) (Bessell $R$-band), Faulkes South $2.0 \mathrm{~m}$ at Siding Spring (Australia) (Bessell $R$-band), Liverpool $2.0 \mathrm{~m}$ at La Palma (Spain) (SDSS $r$-band), and Danish $1.5 \mathrm{~m}$ at La Silla (Chile) (I-band). The CTIO/SMARTS2 bandpasses are close to standard Bessell $V$ and $I$, but sufficiently distinct to be treated separately (Gould et al. 2010). We mark them here by $V_{\mathrm{s}}$ and $I_{\mathrm{s}}$.

Thanks to the public availability of data from the different groups, real-time modelling efforts then showed that the light curve was deviating from a normal Paczyński curve (Paczyński 1986), exhibiting evidence of extended-source effects. The event peaked on that same night (June 15). Because such events are reasonably sensitive to Jupiter-mass planets, as recently shown by Dong et al. (2009), amateur telescopes from the $\mu$ FUN network were alerted, resulting in an excellent coverage of the peak region from 9 different telescopes.

Data reduction has been done using both PSF photometry based on a customized DoPhot package and image subtraction. $\mu \mathrm{FUN}$ telescope images were first reduced using DoPhot. RoboNet/LCOGT data were reduced using an automatic image subtraction package, and reduced again off-line. PLANET telescopes also use image subtraction: at telescope an on-line version called WISIS, based on Alard's ISIS package, then off-line version 3.0 of pySIS (Albrow et al. 2009), based on a numerical kernel (Bramich 2008). SAAO I photometry obtained with pySIS has been checked independently using a DIA package. However, even then the SAAO I data were found to exhibit correlated noise at the peak. Its effect was found to be strong enough to skew the recovered lensing parameters (see Sect. 4). For this reason we decided to omit the light curve from the final event analysis, even though we include it when testing for a potential planetary companion in Sect. 7. SAAO $V$ images were taken well after the light curve peak and therefore do not bring constraints on the event parameters. Bronberg images were re-reduced using the same DIA package. Looking at stars of similar colours as the 
target, a clear airmass effect is generally detected in Bronberg data because there is no filter on the camera. However, in the case of this event, only a clear trend with time was present in the data, instead of the expected airmass trend. As the origin of this trend is unexplained, we could not correct for it and as a result we did not use Bronberg data in the following analysis. CTIO $I_{\mathrm{S}}$ and $V_{\mathrm{s}}$ and LOAO $I$ images were re-reduced off-line using pySIS 3.0, with a slight improvement over DoPhot. CTIO $H$ images were of poor quality and thus discarded. Danish images were reduced using pySIS 3.0; they were also reduced with the DIAPL package from Pych \& Woźniak (Wozniak 2000), with very similar results. Finally, OGLE images were re-reduced with the OGLE pipeline, but with a better resolution reference image and a correctly adjusted centroid.

The final data set before rejection of outliers contains 4389 data points from 11 different telescopes (OGLE I: 1065, CTIO $I_{\mathrm{s}}: 252$, LOAO $I: 159$, CTIO $V_{\mathrm{s}}: 12$, MOA-red: 2555, Canopus $I$ : 44, Perth $I$ : 13 , Skinakas $I$ : 28, Faulkes North $R$ : 12, Faulkes South $R:$ 27, Liverpool $r$ : 59, Danish $I$ : 163).

The shape of the light curve depends on the limb darkening of the source which is different for each photometric band. In the following two figures we plot by solid lines only those that are distinguishable at the peak: the $I$ - and $V_{\mathrm{s}}$-band light curves in Fig. 1, and the $I-, V_{\mathrm{s}^{-}}$and $r$-band light curves in Fig. 2. The curves correspond to the best-fit limb-darkened extended-source model described further and specified in Table 2. The residuals in the lower panels are computed and displayed for their respective light-curve solutions. Dashed lines in these figures correspond to a point-source light curve with the same timescale and impact parameter.

\section{Source properties}

\subsection{Near-infrared colour-magnitude diagram}

The distance to the source and the amount of reddening along the line of sight are uncertainties which always affect the final determination of the properties of the lens-source system. We want to dedicate a short discussion to these issues to justify our adopted values and associated uncertainties and to serve as a reference in our future papers.

Due to the geometry of the galactic bulge with a bar embedded in it, the galactic coordinates of the target give an estimate of the relative position of the source with respect to the galactic centre, if we assume that the source is at the same distance as the majority of the stars in the field. The galactic centre distance itself is adopted from Eisenhauer et al. (2005) as $D_{\mathrm{GC}}=7.62 \pm 0.32 \mathrm{kpc}$ or $\mu_{\mathrm{GC}}=14.41 \pm 0.09$. Rattenbury et al. (2007) give the relative positions of the OGLE-II fields with respect to the field BUL_SC45, which contains Baade's Window $\left(l=1.00^{\circ}, b=-3.88^{\circ}\right)$. As shown by Paczyński \& Stanek (1998), it is safe to assume that the mean distance of stars seen in Baade's Window is similar to the galactic centre distance. Our target's position lies outside of any OGLE-II fields, but close to field BUL_SC20, and this field is at about the same distance as BUL_SC45. We therefore adopt the galactic centre distance modulus as the source distance modulus, but with an increased uncertainty due to the additional involved assumptions, namely $\mu=14.4 \pm 0.2$.

There are several estimates of the reddening in the $K_{\mathrm{s}}$-band at about the position of our target. They range from $A_{K s}=0.28$ (Schultheis et al. 1999) to $A_{K s}=0.46$ (Dutra et al. 2003). Part of the disagreement may come from the patchiness of dust

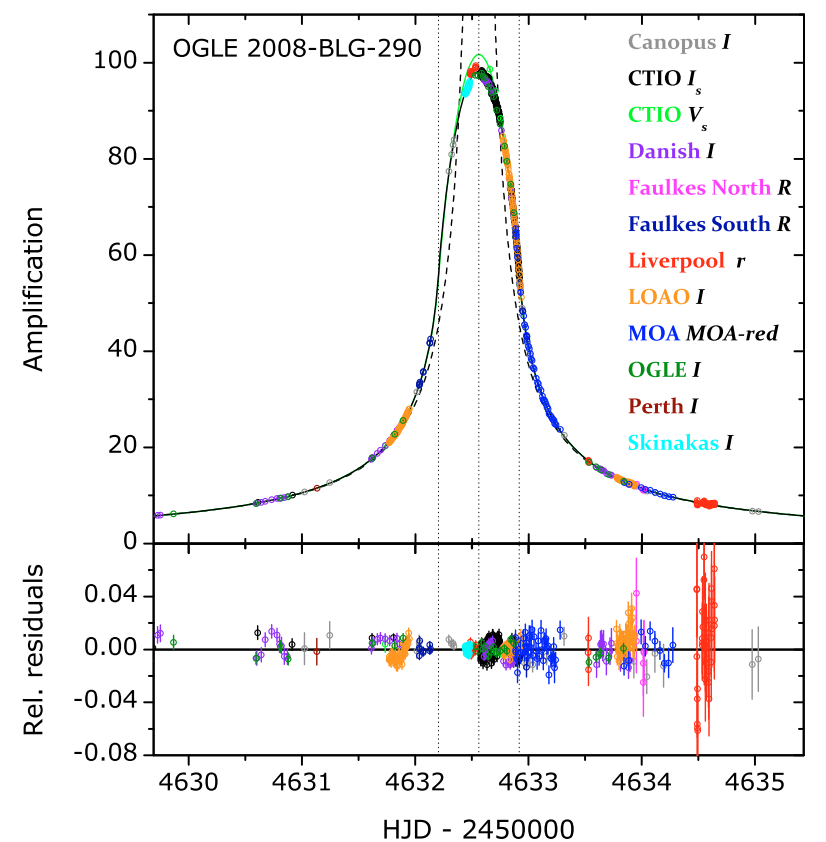

Fig. 1. Upper panel: photometry of microlensing event OGLE 2008BLG-290 in terms of source-flux amplification, as observed by OGLE (olive), MOA (blue), two PLANET sites (Canopus: grey; Perth: wine), three $\mu$ FUN sites (CTIO $1.3 \mathrm{~m}: I_{\mathrm{s}}$ : black, $V_{\mathrm{s}}$ : green; LOAO: orange; Skinakas: cyan), three RoboNet/LCOGT sites (Faulkes North: magenta; Faulkes South: royal blue; Liverpool: red), and one MiNDSTEp site (Danish: violet). The amplification is determined using the limbdarkened source fit with parameters given in Table 2. Plotted lines correspond to the $I$-band (solid black) and $V_{\mathrm{s}}$-band (solid green) light curve solutions, plus a point-source light curve with same impact parameter (dashed black) for comparison. Lower panel: relative flux residuals of the combined data set (the full time span of the analyzed 2008 data runs from $t=4502$ to 4769 ). The vertical dotted lines show the times of source limb entry, closest approach, and limb exit.

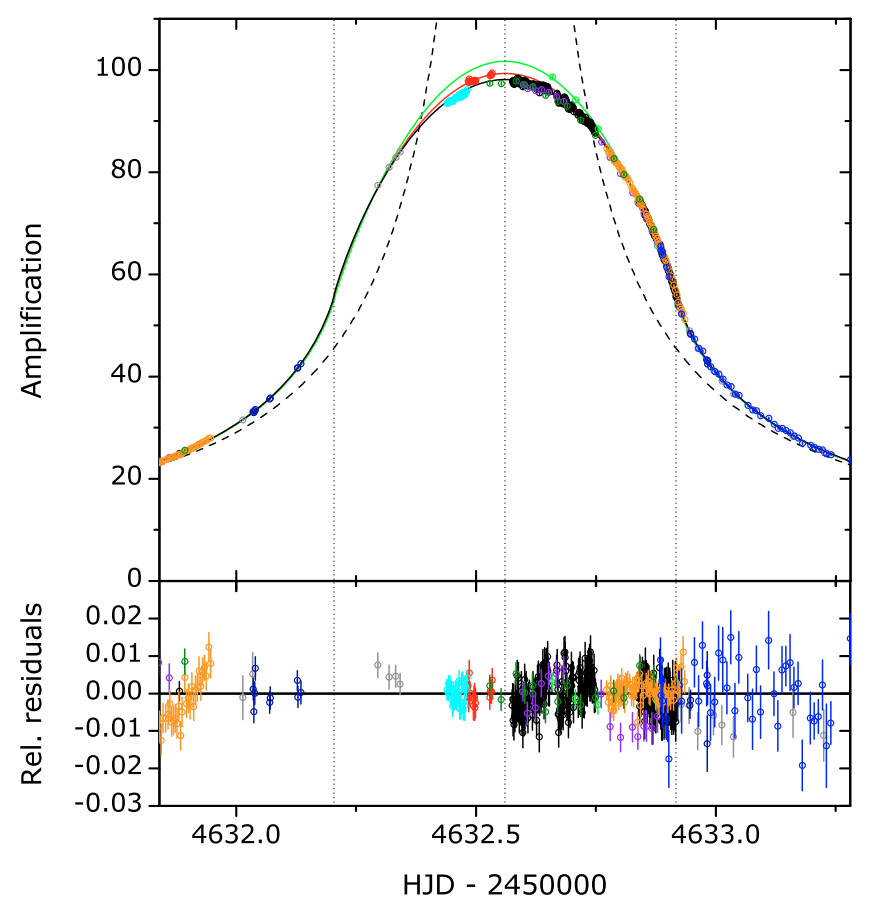

Fig. 2. Zoom of the event peak (June 15, 2008) from Fig. 1. Amplification is plotted in the upper panel; relative residuals in the lower panel. Colours and lines have the same meaning as in Fig. 1, with the added SDSS $r$-band light curve (solid red). 
Table 1. Coordinates and magnitudes of the two stars close to the target position and the 2MASS blend.

\begin{tabular}{lllccc}
\hline \hline Designation & $\alpha(2000)$ & $\delta(2000)$ & $J$ & $H$ & $K_{\mathrm{s}}$ \\
\hline Brighter Northeastern component & $17: 58: 29.362$ & $-27: 59: 21.45$ & $14.291 \pm 0.040$ & $13.369 \pm 0.026$ & $13.042 \pm 0.051$ \\
Fainter Southwestern component & $17: 58: 29.296$ & $-27: 59: 22.00$ & $14.947 \pm 0.054$ & $13.916 \pm 0.038$ & $13.447 \pm 0.023$ \\
2MASS 17582933-2759215 & $17: 58: 29.33$ & $-27: 59: 21.5$ & $13.898 \pm 0.034$ & $12.936 \pm 0.043$ & $12.506 \pm 0.034$ \\
\hline
\end{tabular}

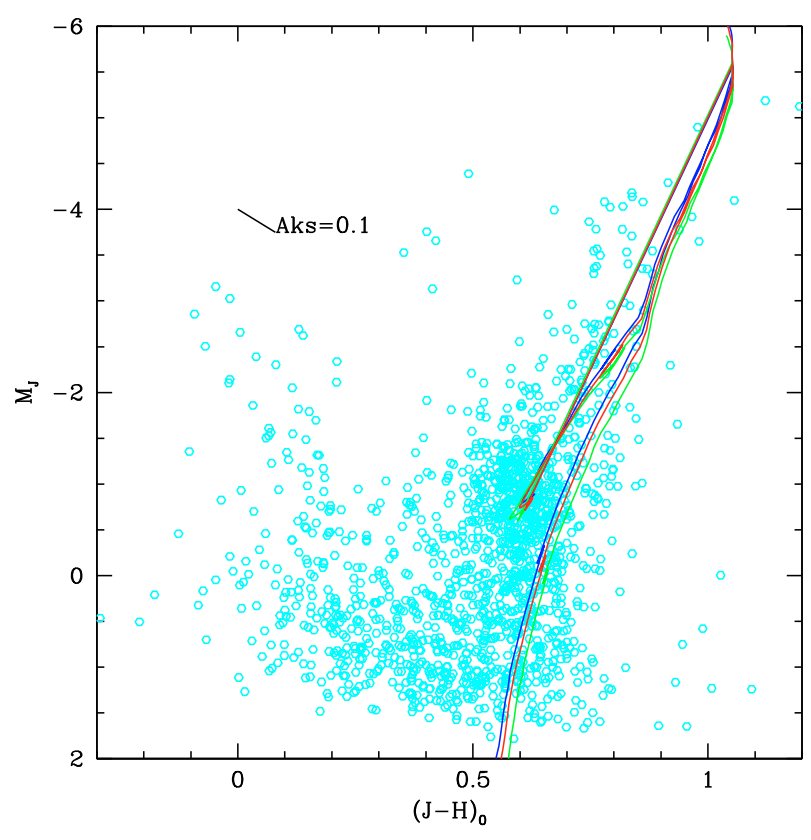

Fig. 3. Colour-magnitude diagram in $J$ and $H$ from IRSF/Sirius stars in a $2.25^{\prime} \times 2.25^{\prime}$ field around OGLE $2008-$ BLG-290 assuming a distance modulus of 14.4, and extinction coefficients of $A_{J}=1.10, A_{H}=0.75$ and $A_{K s}=0.44$. Superimposed are isochrones from Bonatto et al. (2004) for three ages (10, 12.6, $17.8 \mathrm{Gyr}$, from left to right in colour) assuming solar metallicity.

structure, but unfortunately different assumptions about the reddening law also play a role.

Our own estimate is based on IRSF/Sirius photometry of a $7.7^{\prime} \times 7.7^{\prime}$ field containing our target. We use isochrones from Bonatto et al. (2004) based on Padova group models, but directly calibrated for the 2MASS bandpasses. The IRSF/Sirius photometric system, similar to the MKO system (Tokunaga et al. 2002), is close to the 2MASS system, but we calibrated the photometry using 2MASS stars in the same field to ensure coherence.

We restrict the fitting region to 300 pixels around the target $\left(2.25^{\prime} \times 2.25^{\prime}\right)$ to avoid differential extinction. This is large enough to form well-defined colour-magnitude diagrams (hereafter CMD), where the red giant clump (hereafter RC) is easily identified. This is not the case when using only 2MASS, because the brighter limiting magnitude cuts off part of the clump. An example of such a CMD is displayed in Fig. 3.

Although the position of the clump could in principle give an estimate of its distance, in practice variations in age and metallicity do not allow an accurate determination. Adopting a $10 \mathrm{Gyr}$ isochrone and a solar metallicity, we get the following estimates of the near-infrared extinction and reddening law:

$A_{K s}=0.44$

$A_{J}=2.5 \times A_{K s}$

$A_{H}=1.7 \times A_{K s}$.
Note that the corresponding reddening law in $J$ differs from what Nishiyama et al. (2006) found based on the IRSF/Sirius galactic bulge survey $\left(A_{J}=3.0 \times A_{K s}\right)$, obtained with the same telescope and instrumentation. This possibly reflects variations of the reddening law in different directions of the galactic bulge.

The target, which appears as a double star in the OGLE finding chart, is well separated by PSF photometry of the IRSF/Sirius image. The accurate coordinates and magnitudes of the two components are given in Table 1, together with the corresponding values for the single object in the 2 MASS catalogue.

Although the 2MASS flags do not indicate any blending, the coordinates and magnitudes correspond well to the blend of the two stars. The microlensed target is the fainter southwestern component and, after extinction correction and conversion to the Bessell \& Brett photometric system (Bessell \& Brett 1988), it has $K_{0}=13.05$ and typical colours of a K4 giant star $\left((J-K)_{0}=0.87,(H-K)_{0}=0.13\right)$. Such a star is predicted to have colours of $(V-I)_{0}=1.50$ and $(V-K)_{0}=3.26$, so we expect $V_{0}=16.3$ and $I_{0}=14.8$.

Estimating the uncertainties of the measured near-infrared extinctions is not straightforward, because the present method mixes hypotheses about distance, age, metallicity with actual measurements by isochrone fitting. In the next paper of this series (Fouqué, in prep.) on MOA 2009-BLG-411, a refined method will be introduced, using reddening law, clump absolute magnitudes and distance hypothesis to simultaneously fit isochrones to near-infrared and visible CMDs and derive a coherent source size. We applied this new technique to the present data set to estimate the uncertainties in our measured extinctions. We found differences of 0.06, 0.13 and 0.05 in $A_{J}, A_{H}$ and $A_{K s}$, respectively. We therefore adopt an uncertainty of 0.1 in the extinction measurement for each near-infrared band.

\subsection{Visible CMD}

A colour-magnitude diagram allows an estimate of the dereddened magnitude and colour of the source, by comparison with the observed position of the red giant clump, if one assumes that both suffer the same amount of extinction. The CTIO $1.3 \mathrm{~m}$ telescope obtained the uncalibrated colour-magnitude diagram displayed in Fig. 4 (instrumental magnitudes), from which we read a magnitude shift in $I$ of $0.56 \pm 0.1$ for the source compared to the RC, and a colour shift of $0.37 \pm 0.1$.

For the mean absolute magnitude of the clump, we adopt the Hipparcos value as given by Stanek \& Garnavich (1998), $M_{\mathrm{I}}=-0.23 \pm 0.03$ and for the mean colour $(V-I)_{0}{ }^{\mathrm{RC}}=1.00 \pm$ 0.05 . With our adopted distance to the field, $\mu=14.4 \pm 0.2$, this gives $I_{0}^{\mathrm{RC}}=14.17 \pm 0.2$. So our source is predicted to have $I_{0}^{\mathrm{S}}=14.73 \pm 0.23$ and $(V-I)_{0}{ }^{\mathrm{S}}=1.37 \pm 0.11$, in good agreement with the values obtained before from the near-infrared CMD.

From magnitude and colour, and using the recent revision of surface-brightness-colour relations (hereafter SBC) in $I,(V-I)$ published by Kervella \& Fouqué (2008), we get an estimate of the angular source radius $\theta_{*}$ in $\mu$ as of:

$\log \theta_{*}=-0.2 I_{0}+0.4895(V-I)_{0}-0.0657(V-I)_{0}^{2}+3.198$. 
Table 2. Main parameters of the best-fitting point-lens limb-darkened source model, using the linear limb-darkening law.

\begin{tabular}{|c|c|}
\hline Parameter & Value \& Errors \\
\hline$t_{0}(\mathrm{HJD}-2450000)$. & $4632.56037(-0.00027+0.00019)$ \\
\hline$u_{0} \ldots \ldots \ldots \ldots \ldots$ & $0.00276(-0.00020+0.00021)$ \\
\hline$t_{\mathrm{E}}($ days $) \ldots \ldots \ldots$ & $16.361(-0.077+0.071)$ \\
\hline$\rho_{*} \ldots \ldots \ldots \ldots \ldots$ & $0.02195(-0.00011+0.00010)$ \\
\hline$u_{V_{\mathrm{s}}} \ldots \ldots \ldots \ldots \ldots$ & $0.773(-0.011+0.014)$ \\
\hline$u_{r} \ldots \ldots \ldots \ldots \ldots$ & $0.642(-0.058+0.087)$ \\
\hline$u_{R} \ldots \ldots \ldots \ldots \ldots$ & $0.617(-0.046+0.071)$ \\
\hline$u_{\mathrm{MOA}} \ldots \ldots \ldots \ldots$ & $0.593(-0.037+0.041)$ \\
\hline$u_{I_{\mathrm{s}}} \ldots$ & $0.569(-0.007+0.005)$ \\
\hline$u_{I} \ldots \ldots \ldots \ldots \ldots$ & $0.528(-0.008+0.009)$ \\
\hline$\chi^{2} /$ d.o.f........... & $2317.7 /(2049-34)=1.150$ \\
\hline
\end{tabular}

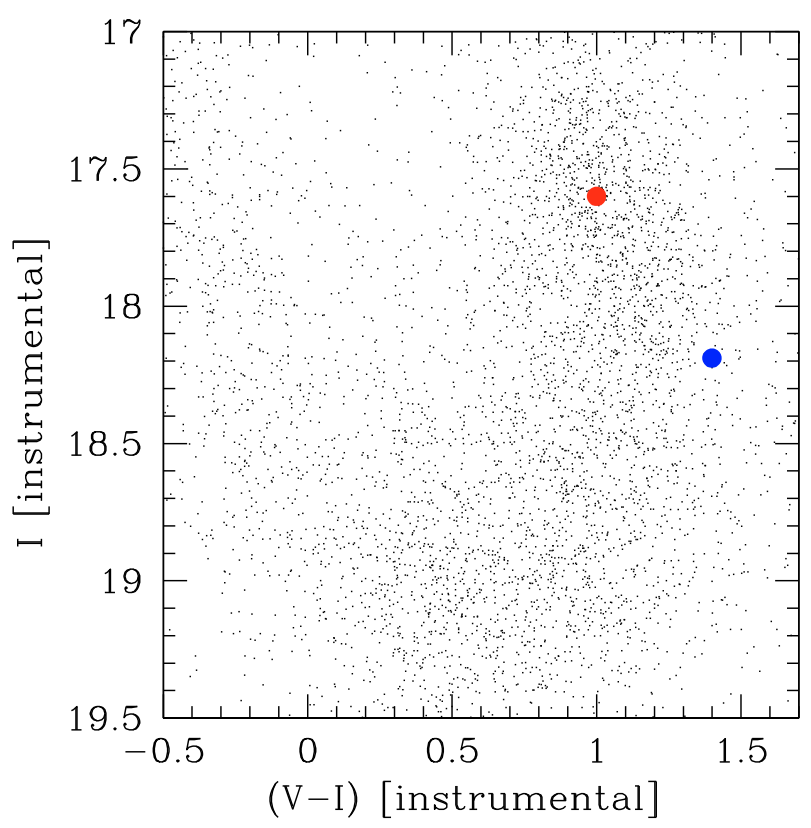

Fig. 4. Colour-magnitude diagram in $I_{\mathrm{s}}$ and $V_{\mathrm{s}}$ from CTIO $1.3 \mathrm{~m}$ telescope. The blue disk shows the source position, while the red one marks the centroid position of the red giant clump.

The uncertainty of this estimate is 0.0238 , so adding quadratically the uncertainty in magnitude and colour gives $\theta_{*}=6.3 \pm 1.1 \mu$ as.

However, this source is probably a giant star, and these SBC relations have been calibrated for dwarfs and sub-giants. In order to check if this introduces a systematic uncertainty in our measurement of the angular radius, we use the Groenewegen (2004) SBC relation specifically designed for giants:

$$
\log \theta_{*}=-0.2 K_{0}+0.045(V-K)_{0}+3.283 \text {. }
$$

The uncertainty of this estimate is similar at 0.024 , so adding quadratically the uncertainty in the magnitude $K_{0}=13.05 \pm 0.06$ and estimated colour $(V-K)_{0}=3.1 \pm 0.2$ gives $\theta_{*}=6.5 \pm 0.4 \mu \mathrm{as}$, in agreement with the previous estimate. At the adopted source distance, this translates into a linear radius of $R_{*}=10 R_{\odot}$, typical of a $\mathrm{K}$ giant. Please note that in the estimate using the visible $\mathrm{CMD}$, the angular radius depends on the adopted source distance, while the linear radius does not. Conversely, in the nearinfrared method, the angular radius depends very little on the adopted source distance while the linear radius does. The agreement between both values shows that our adopted source distance is correct.
Using the dereddened colours and, for instance, Houdashelt et al. (2000) tables, we estimate the effective temperature of the source star to be about $4200 \mathrm{~K}$, corresponding to a $\mathrm{K} 3$ giant. In order to estimate an uncertainty of this value, we repeated the whole procedure adopting plausible different values for the clump distance, its colour and absolute magnitude, and measured the shift in colour and magnitude of the source with respect to the centroid of the clump using the OGLE-III photometric catalogue. The difference in colours that we get using these new values corresponds to a difference in temperature of about $100 \mathrm{~K}$. We therefore adopt $4200 \pm 100 \mathrm{~K}$ as our estimate of the photometric temperature of the source star.

Using this value, we looked at Marigo et al. (2008) isochrones for a model star with similar characteristics to ours. Two routes are used to derive the star's luminosity: on one hand, from $K_{0}=13.05$, distance modulus (14.4) and model bolometric correction in $K(2.36)$, we get $M_{\text {bol }}=1.01$ and $\log L / L_{\odot}=1.48$; on another hand, we use $T_{\text {eff }}$ and our estimate of the star's radius, to get $\log L / L_{\odot}=1.45$. We note that different modelers still use different values of the bolometric magnitude of the Sun (4.72 for Houdashelt et al. 2000; vs. 4.77 for Marigo et al. 2008). We find that an old star $(12.7 \mathrm{Gyr})$, slightly metal-rich $(Z=0.03)$ gives a red giant of 1 solar mass and $\log g=2.5$, with such characteristics.

\section{Data modelling: noise model}

From the original data set, we remove data points in OGLE and MOA older than date 4490 or newer than 4770 . This corresponds to selecting the whole 2008 observing season. We verified that this does not change the resulting fit parameters. The reason for this cut is two-fold: the planetary deviation search is very demanding in terms of CPU time, so reducing the number of useful points helps; moreover, the number of data points in the baseline before 4490 is quite large, and any slight error in the photometric error estimate may bias the fit.

We then proceed to remove outliers and rescale photometric error bars in a consistent way: the rescaling factor is computed by forcing $\chi^{2} /$ d.o.f. $\simeq 1$ for each telescope data set independently, and after rescaling, any point lying at more than $3 \sigma$ is removed. This slightly changes the estimate of $\chi^{2}$ and thus the rescaling factor. After a few iterations, the process converges. Care was taken not to remove any potential planet-caused outliers. The number of outliers varies between 1 and 6 per telescope.

We return briefly to the SAAO I-band light curve. Inspecting the residuals of the microlensing fit, we find they are mutually correlated rather than randomly distributed, as mentioned earlier in Sect. 2. Near the event peak clusters of points with positive residuals alternate with negative-residual clusters. Despite its small amplitude, the effect is strong enough to influence the recovered lensing parameters. Adding the SAAO light curve to the twelve other curves results in a lower impact parameter and shorter source-radius crossing time, both of which in turn influence the limb-darkening measurement. The small-amplitude abrupt shifts in the SAAO residuals during the crossing are most probably a systematic artifact in the data (see also Sects. 7 and 8). We therefore decided not to include this light curve in the subsequent event analysis.

In the case of MOA data, several measurements close to the peak were saturated and therefore were eliminated. For this particular telescope, 51 data points were removed a priori or as outliers. For OGLE data, we quadratically added 0.003 mag to all photometric errors, in order to avoid too small errors at the peak, 
which correspond to Poisson noise at these bright magnitudes, but do not reflect systematic errors.

The final total number of fitted data points amounts to 2049 in 12 light curves observed in 6 different photometric bands (see Fig. 1).

\section{Limb darkening of the source star}

\subsection{Limb-darkening measurement}

As expected, a single-lens point-source model results in a high $\chi^{2} /$ d.o.f. $\sim 40$, which obviously rejects this simple model. A uniform extended-source model provides us with a first working set of parameters; however, the fit still has a high $\chi^{2} /$ d.o.f. $\sim 3.6$. At this stage the residuals of the fit show a symmetric pattern around the peak of the light curve, the signature of limb darkening. We thus add linear limb darkening to the source model. This is described here by

$I(r) \propto 1-u\left(1-\sqrt{1-r^{2}}\right)$,

where $r$ is a radial coordinate running from 0 at disk centre to 1 at limb, and $u$ is the linear limb-darkening coefficient (hereafter LLDC, e.g., Claret 2000).

The adopted event parametrization involves the following microlensing quantities: $t_{0}$ (time of closest approach), $u_{0}$ (impact parameter in units of the Einstein ring radius $\left.\theta_{\mathrm{E}}\right), t_{\mathrm{E}}$ (crossing time of $\left.\theta_{\mathrm{E}}\right), \rho_{*}$ (source radius in units of $\theta_{\mathrm{E}}$ ) and two annual parallax parameters $\pi_{\mathrm{E}}$ and $\psi$ (see Sect. 6) common to all data sets; plus light-curve-specific parameters: baseline and blending fluxes, as well as the LLDC $u$ for the respective photometric band. The main parameters of the set best fitting the data $\left(\chi^{2} /\right.$ d.o.f. $\left.=1.15\right)$ and their errors are given in Table 2. The limb-darkening coefficients correspond to the involved six distinct photometric bands: CTIO/SMARTS $2 V_{\mathrm{s}}$, SDSS $r, R$, MOAred, CTIO/SMARTS $2 I_{\mathrm{S}}$, and $I$, in order of increasing effective wavelength.

Based on the final model, we find that the source-radius transit time is $\rho_{*} t_{\mathrm{E}} \simeq 0.36 \mathrm{~d}$; the time (in terms of $t=$ HJD-2 450000 ) at which the lens starts to transit the source disk $t_{\text {entry }} \simeq 4632.204 \mathrm{~d}$, while the exit time is $t_{\text {exit }} \simeq 4632.917 \mathrm{~d}$. The peak magnification achieved in the $I$-band is $\sim 97$, in the $V_{\mathrm{s}}$-band $\sim 102$. The source $I$-band magnitude in the OGLE-III photometric database is 17.26 , so the source star temporarily brightened to magnitude 12.3 due to the lens passing in the foreground.

Individual telescopes constrain the measured limb darkening unequally, because of their different coverage of the causticcrossing peak. As a result, Perth, Skinakas and Faulkes-North do not contribute, Faulkes-South very weakly (closest points to the peak are just outside the limb) and Liverpool only weakly. Hence, the obtained limb darkening in the $R$-band (Faulkes telescopes) and the $r$-band (Liverpool) is poorly constrained. In the $V_{\mathrm{s}^{-}}$and $I_{\mathrm{S}}$-bands, the LLDCs rely on the respective CTIO light curves. With the MOA-red band result determined by MOA data, only the $I$-band result is constrained by 4 different telescope data sets (Danish, LOAO, OGLE, Canopus).

In order to test for consistency we fit the event data again, using common lensing parameters but leaving the limb darkening independent for each of the 12 data sets. Checking the $I$-band LLDCs shown in Fig. 5, we find that even though the Canopus value is consistent with the others at the $3-\sigma$ level, the scatter of the values exceeds individual errors as well as the error obtained from the straightforward fit in Table 2 . In the light of this finding, the small error on $u_{I}$ in Table 2 is not really the result of

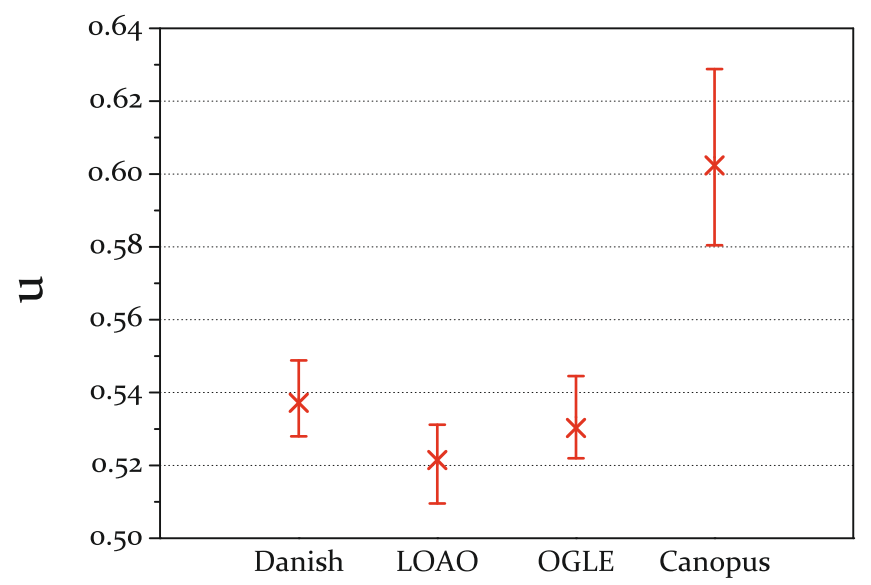

Fig. 5. Linear limb-darkening coefficients in the $I$-band for 4 different telescopes when fitting the limb darkening independently for each data set. We determine the $I$-band measurement as the weighted mean of these values, $u_{I}=0.539 \pm 0.033$.

an excellent agreement between individual light curves. Rather, it is a statistical artifact obtained by the competing higher- $u$ value Canopus and the lower- $u$-value-favouring others. Possible causes of this discrepancy include slightly different filters, detector or atmosphere response, light curve coverage, secondary amendments of the source or lens models, but also noise models for each telescope. To get a more realistic result under these circumstances, we determine the I-band LLDC from the values in Fig. 5. The weighted mean of the individual values with the statistical error given by their scatter yields $u_{I}=0.539 \pm 0.033$.

\subsection{Comparison with model atmospheres}

We obtain the response functions of the individual telescopes by combining their filter transmission curves and their CCD quantum efficiency curves. We then use the response functions to compute theoretical values of the LLDCs, based on Kurucz's ATLAS9 atmosphere models (Kurucz 1993a,b, 1994). To get the LLDC for a particular theoretical limb-darkening profile we use the radially integrated fit method described in Heyrovský (2007) rather than the 11-point fit from Claret (2000), for reasons described in the former reference. We compute the LLDC values for a sub-grid of Kurucz's model grid based on the source properties inferred in Sect. 3, with effective temperature $T_{\text {eff }}$ ranging from $4000 \mathrm{~K}$ to $5000 \mathrm{~K}$, metallicity $[\mathrm{Fe} / \mathrm{H}]$ from 0 to +0.3 , surface gravity $\log g$ from 2.0 to 3.0 , and microturbulent velocity fixed at $v_{\mathrm{t}}=2 \mathrm{~km} \mathrm{~s}^{-1}$.

The comparison of the measured LLDC in the 6 bands to theoretical model predictions is presented in Fig. 6. Judging first the agreement between the bands, we point out that the obtained values decrease with increasing effective wavelength, in agreement with theoretical expectations. Second, we note that within each band the measured values point to a similar effective temperature of the source, with all measurements being mutually compatible within their error bars. This leads to an LLDC-based initial estimate of the effective temperature of the source star, with the most accurate $V_{\mathrm{s}}$ and $I_{\mathrm{s}}$ measurements indicating $4750 \mathrm{~K}$. The value is in rather poor agreement with the photometric estimate $4200 \mathrm{~K}$ derived in Sect. 3.2. We note that the theoretical Claret (2000) coefficients would lead to an even larger discrepancy, with the $I$-band measurement indicating an effective temperature higher than $5500 \mathrm{~K}$. 
P. Fouqué et al.: OGLE 2008-BLG-290, a microlensed galactic bulge K Giant

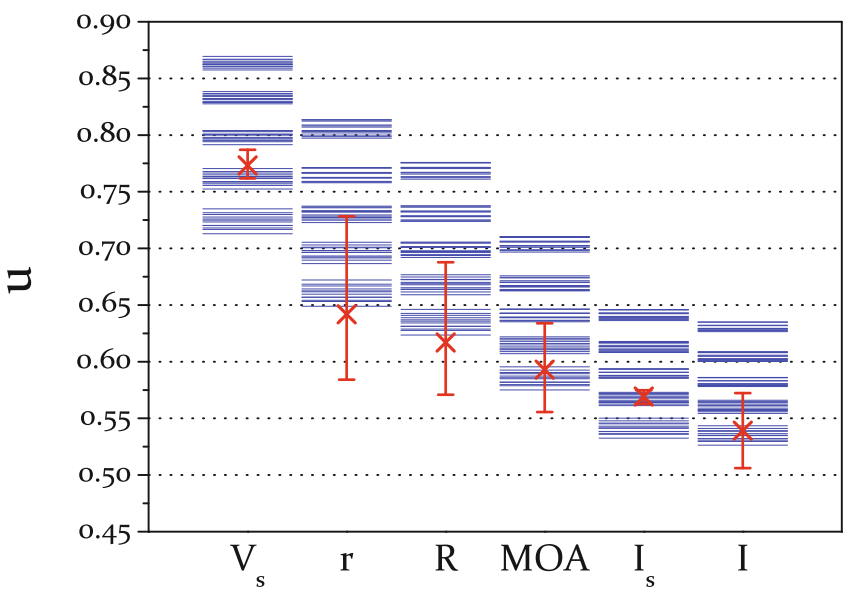

Fig. 6. Linear limb-darkening coefficients of the K-giant source star in CTIO/SMARTS2 $V_{\mathrm{s}}-$, SDSS $r-, R-$, MOA-red, CTIO/SMARTS2 $I_{\mathrm{s}}-$, and $I$-bands, compared to theoretical model atmosphere predictions (blue) for different effective temperatures (five broad bands in $250 \mathrm{~K}$ steps from $4000 \mathrm{~K}$ at top to $5000 \mathrm{~K}$ at bottom), different metallicities (from $[\mathrm{Fe} / \mathrm{H}]=0.3$ at top to 0.0 at bottom), and different surface gravities (finest structure with $\log g=2.0,2.5,3.0$ ) at each temperature.

Comparing measured LLDCs with corresponding values computed from model atmospheres has its potential pitfalls (Heyrovský 2003, 2007). Simple analytical limb-darkening laws, and the linear law in particular, often do not describe theoretical limb-darkening profiles sufficiently accurately. Such analytical approximations do not even conserve the flux of the approximated profile with the required precision. To avoid this problem at least partly, we compare our measured LLD profiles directly with the theoretical profiles of the Kurucz models rather than with their linear limb-darkening approximations.

We denote by $I_{\text {Measured }}$ our measured intensity profile given by Eq. (6) with the measured LLDC $u$, normalizing it to unit rms intensity. For each theoretical profile from the considered subgrid we then compute a scale factor yielding the best agreement with $I_{\text {Measured }}$ and mark the rescaled theoretical profile $I_{\text {Kurucz }}$. In Fig. 7 we plot the obtained difference curves $I_{\text {Kurucz }}-I_{\text {Measured }}$ as a function of radial position on the stellar disk for our most precise measurements, i.e., the $V_{\mathrm{s}^{-}}$and $I_{\mathrm{s}}$-band limb darkening. From the plot one can already distinguish by eye the agreement with Kurucz profiles of different effective temperature, metallicity, and surface gravity. For instance, all the $4750 \mathrm{~K}$ profiles agree with the measured $V_{\mathrm{s}}$ profile within $1.3 \%$ of the rms intensity from disk centre out to the limb. Nevertheless, in the case of the measured $I_{\mathrm{s}}$ profile it is hard to judge by eye which group of model profiles agrees better. The $4500 \mathrm{~K}$ models are preferred near the centre, but closer to the limb their accuracy drops earlier than that of the $4750 \mathrm{~K}$ models.

To obtain a quantitative measure of agreement we compute the relative residual $\delta=$ $\sqrt{\int\left(I_{\text {Kurucz }}-I_{\text {Measured }}\right)^{2} \mathrm{~d} r} / \sqrt{\int I_{\text {Measured }}^{2} \mathrm{~d} r}$, defined as the rms difference in units of rms intensity. The values of $\delta$ corresponding to the profiles in Fig. 7 are presented graphically in Fig. 8. Each point in the left (right) grid corresponds to a Kurucz $V_{\mathrm{s}}$-band $\left(I_{\mathrm{s}}\right.$-band) profile with the corresponding $T_{\text {eff }}$, $[\mathrm{Fe} / \mathrm{H}]$, and $\log g$ values as the coordinates. The colour of the point indicates the value of $\delta$ as shown in the colour bar. The black point singles out the best agreeing model with the relative residual $\delta=\delta_{\text {MIN }}$ given above the grid. Clearly $T_{\text {eff }}=4750 \mathrm{~K}$ is strongly favoured in both bands, validating our previous finding.
As for the other parameters, there is a weaker preference for higher $[\mathrm{Fe} / \mathrm{H}]$ and higher $\log g$.

In order to get an error bar estimate on $T_{\text {eff }}$, we perform a similar analysis for profiles with LLDC values given by the upper and lower error bars in either band from Table 2 . We find that the lower limits on $u_{V_{\mathrm{s}}}$ and $u_{I_{\mathrm{s}}}$ both agree best with $4750 \mathrm{~K}$ models, while the upper limits on both agree best with $4500 \mathrm{~K}$ models. Based on these results and the change of $\delta$ with $T_{\text {eff }}$ in Fig. 8 we conclude that our limb-darkening measurement yields a temperature estimate of the source star $T_{\text {eff }}=4700_{-200}^{+100} \mathrm{~K}$, obtained by comparison with the limb darkening of Kurucz's models.

The LLDC measured from our analysis for the $I$-band is presented in Table 3 and compared to model LLDC predictions for the given stellar parameters, together with values for similar giants resolved by microlensing, namely EROS 2000-BLG5 from Fields et al. (2003) as reported in Yoo et al. (2004), OGLE 2003-BLG-238 from Jiang et al. (2004) and OGLE 2004BLG-254 from revised fits in Heyrovský (2008), which solve discrepancies noted in Cassan et al. (2006). Unfortunately, the listed effective temperatures are based on photometric estimates for all events but OGLE 2004-BLG-254, for which the spectroscopic measurement $(4100 \mathrm{~K})$ disagrees with the photometric one (4500 K) given in the Table. OGLE 2008-BLG-290 appears to be a twin of EROS 2000-BLG-5, while OGLE 2003-BLG-238 and OGLE 2004-BLG-254 are slightly hotter.

For the present event, the agreement between temperatures estimated from colours and from limb-darkening coefficients is not satisfactory $\left(4200 \pm 100\right.$ vs. $4700_{-200}^{+100} \mathrm{~K}$, respectively). Unfortunately, we do not have a spectroscopic estimate of $T_{\text {eff }}$, but it is not unusual that photometric and spectroscopic estimates disagree by several hundred $\mathrm{K}$ in cool giants. We refer to Affer et al. (2005) and Fulbright et al. (2006) for detailed discussions about this discrepancy.

One way to solve the disagreement is to assume that our target suffers more extinction than the red clump in the same region. Let us check what it would imply if the source star suffers 0.1 mag more extinction in the $K_{\mathrm{s}}$-band than the clump. According to our adopted reddening law in the near-infrared (Eqs. (2) and (3)), the source star would be at $M_{\mathrm{J}}=-0.73$ and $(J-H)_{0}=0.66$, inside the red clump. Adopting typical reddening law ratios of 10 and 5.6 between respectively $A_{V}$ and $A_{I}$ on one side, and $A_{K s}$ on the other side, the source star brightens by $0.56 \mathrm{mag}$ in $I$ and becomes bluer by $0.44 \mathrm{mag}$ in $(V-I)$, explaining the observed shifts in the visible CMD with respect to the red clump centroid not because the star is redder and fainter, but because it suffers more extinction.

As the typical temperature of Bulge red clump giants is about $4750 \mathrm{~K}$ (Hill, personal communication), this would approximately reconcile the effective temperature deduced from the LD measurements with the photometric temperature.

Obviously, this would slightly decrease (by about 5\%) the angular radius of the source obtained from the surface brightness colour relations (Eqs. (4) and (5)) and accordingly all the dependent parameters, but the exact change depends on the visible to near-infrared extinction ratios, which are not well known (e.g. Nishiyama et al. 2008).

\section{Lens properties}

With the angular Einstein radius being related to the angular source radius $\theta_{*}$ as $\theta_{\mathrm{E}}=\theta_{*} / \rho_{*}$, we find $\theta_{\mathrm{E}}=300 \pm 20 \mu$ as. This enables us to calculate the relative lens-source proper motion: $\mu=\theta_{\mathrm{E}} / t_{\mathrm{E}}=6.7 \pm 0.4 \mathrm{mas} / \mathrm{yr}$. The relative transverse velocity $v_{\perp}$ 

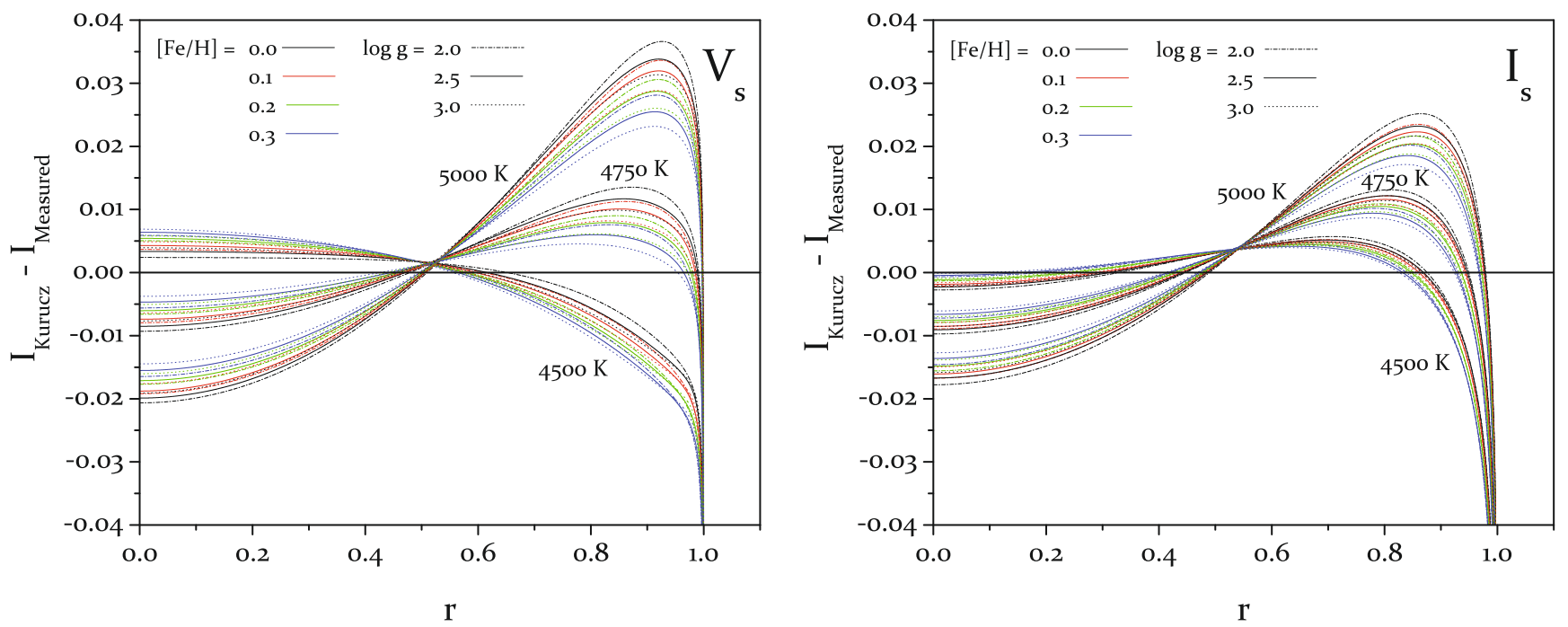

Fig. 7. Comparison of the measured $V_{\mathrm{s}}$-band (left panel) and $I_{\mathrm{s}}$-band (right panel) limb-darkening profiles with corresponding theoretical limbdarkening profiles from Kurucz's ATLAS9 model-atmosphere grid: absolute difference $I_{\text {Kurucz }}-I_{\text {Measured }}$ as a function of radial position $r$ on the stellar disk. $I_{\text {Measured }}$ is normalized to unit rms intensity; each theoretical profile $I_{\text {Kurucz }}$ is scaled vertically to minimize the rms difference. The three broad bands formed by the curves correspond to three effective temperatures $T_{\text {eff }}$, colours indicate four metallicities [Fe/H], and line types mark three surface gravities $\log g$.
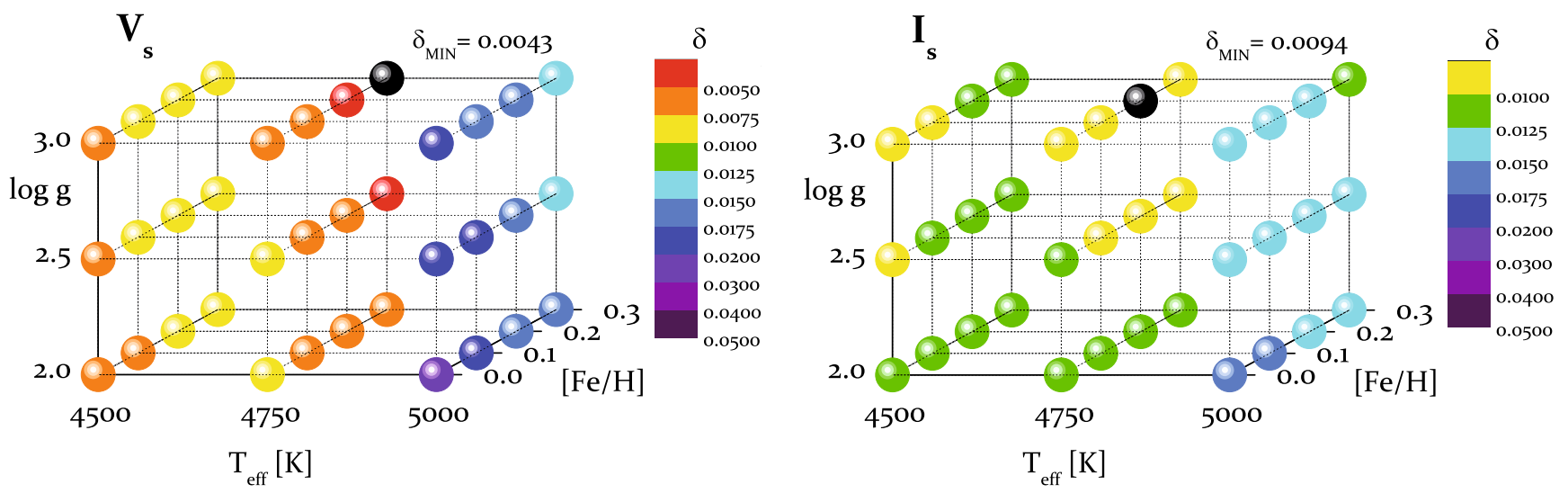

Fig. 8. Agreement between the measured $V_{\mathrm{s}}$-band (left panel) and $I_{\mathrm{s}}$-band (right panel) limb-darkening profile and Kurucz limb-darkening profiles, as indicated by the colour-coded relative residual $\delta$. With the normalization used in Fig. 7, values of $\delta$ are equal to the rms of each of the difference curves in Fig. 7. The three Cartesian coordinates in the plotted grid correspond to the three main stellar-atmosphere parameters in the Kurucz model grid, $T_{\text {eff }},[\mathrm{Fe} / \mathrm{H}]$, and $\log g$. The best-agreeing model is marked by the black dot and has the value $\delta_{\text {MIN }}$ given above the plot.

Table 3. Photometric temperatures and I-band limb-darkening coefficients of K Bulge giants for OGLE 2008-BLG-290 and other published microlensing events with source stars of similar spectral type.

\begin{tabular}{|c|c|c|c|c|c|c|}
\hline \multirow[t]{2}{*}{ Event } & \multicolumn{4}{|c|}{ Source characteristics } & \multirow{2}{*}{$\begin{array}{c}\text { Measured LLDC } \\
u_{I}\end{array}$} & \multirow{2}{*}{$\begin{array}{c}\text { ATLAS LLDC } \\
u_{I}\end{array}$} \\
\hline & Type & $T_{\text {eff }}$ & $\log g$ & {$[\mathrm{Fe} / \mathrm{H}]$} & & \\
\hline EROS 2000-BLG-5 & K3 III & $4200 \mathrm{~K}$ & 2.3 & 0.3 & $0.54^{a}$ & 0.62 \\
\hline OGLE 2003-BLG-238 & K2 III & $4400 \mathrm{~K}$ & 2.0 & 0.0 & $0.58 \pm 0.06$ & 0.59 \\
\hline OGLE 2004-BLG-254 & K3 III & $4500 \mathrm{~K}$ & 2.0 & 0.3 & $0.62 \pm 0.07$ & 0.59 \\
\hline OGLE 2008-BLG-290 & K3 III & $4200 \mathrm{~K}$ & 2.5 & 0.2 & $0.539 \pm 0.033$ & 0.61 \\
\hline
\end{tabular}

Notes. ${ }^{(a)}$ One-parameter fit cannot properly describe the LD profile: see Sect. 5 for details.

between lens and source at the lens distance then follows from $v_{\perp}=D_{\mathrm{L}} \mu$.

From the value of $\theta_{\mathrm{E}}$ in mas and $D_{\mathrm{S}}$ in kpc, we obtain a constraint on the lens mass $M_{\mathrm{L}}$ in solar mass units as (Dominik 1998):

$M_{\mathrm{L}}(x)=\frac{\theta_{\mathrm{E}}^{2} D_{\mathrm{S}}}{8.144} \frac{x}{1-x}$, where $x=D_{\mathrm{L}} / D_{\mathrm{S}}$, which for $D_{\mathrm{S}}=7.6 \mathrm{kpc}$ and $\theta_{\mathrm{E}}=0.30$ mas gives:

$M_{\mathrm{L}}(x)=0.084 \frac{x}{1-x} M_{\odot}$.

In principle, a measurement of the source size in both Einstein radius and physical units, as well as the measurement of parallax parameters completely determine the lens location (given the source distance $D_{\mathrm{S}}$ ). Indeed, similarly to Eq. (7), a measured 

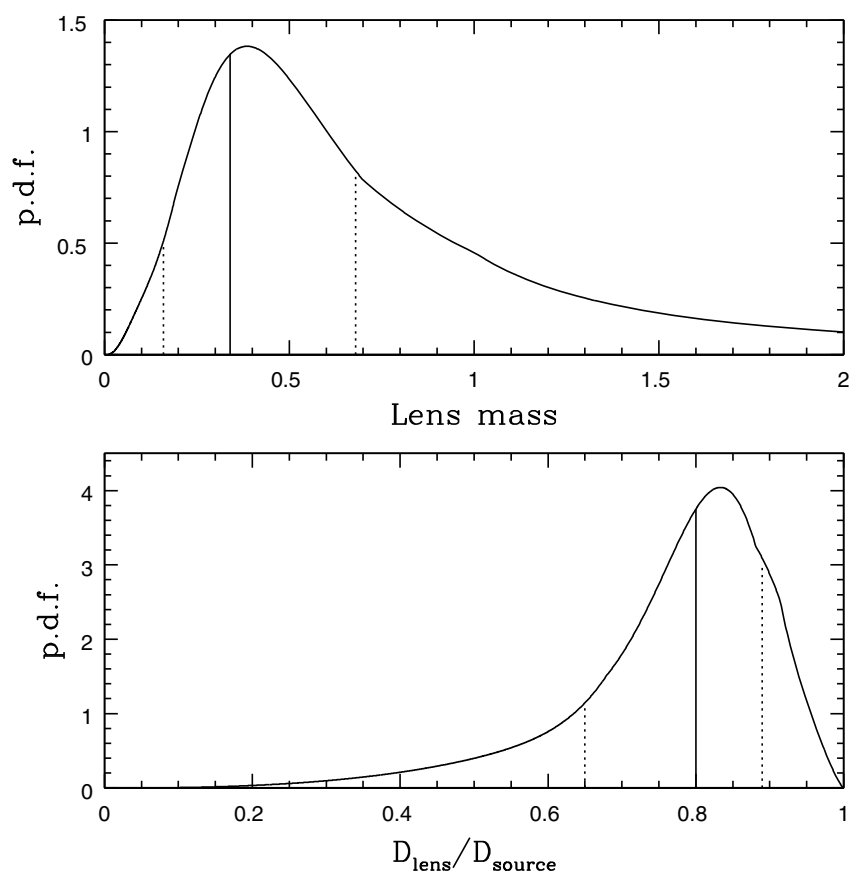

Fig. 9. Probability densities for the lens mass $M_{\mathrm{L}} / M_{\odot}$ and the lens distance $D_{\mathrm{L}}$ from the adopted Galaxy model. Vertical lines correspond to median, first and last quartiles.

parallax parameter would provide a relation between lens mass and $x$, but unfortunately the event is too short (16 days $\ll 1$ year) to provide a measurement of the orbital parallax, or even to give reasonable limits. For a similar event (duration $t_{\mathrm{E}} \simeq 13$ days, source size $\rho_{*} \simeq 0.06$ and very small impact parameter $u_{\circ}$ ), Yoo et al. (2004) only obtained a marginal parallax measurement as well.

We then use estimates of the physical parameters, following Dominik (2006) and assuming his adopted Galaxy model. The event time-scale $t_{\mathrm{E}}=16.4$ days and the angular Einstein radius $\theta_{\mathrm{E}}=0.3$ mas provide us with probability densities for the lens mass $M_{\mathrm{L}}$ and the lens distance $D_{\mathrm{L}}$, which are shown in Fig. 9.

From these, we find a median lens mass $M_{\mathrm{L}} \sim 0.3 M_{\odot}$, a median projected relative velocity $v_{\perp} \sim 190 \mathrm{~km} \mathrm{~s}^{-1}$, and a median lens distance $D_{\mathrm{L}} \sim 6.0 \mathrm{kpc}$ for an adopted source distance of $D_{\mathrm{S}}=7.6 \mathrm{kpc}$. The lens is inferred to reside in the galactic bulge, with $60 \%$ probability.

\section{Possible planetary deviations}

In this section we include even the 120 points of the SAAO $I$-band data in the analysis to test whether the overall residuals may harbour the weak signature of a planetary companion of the lens. The single-lens extended-source model with fitted limb-darkening coefficients then has a $\chi^{2}$ of 2485.7 for 2169 data points (after rescaling error bars). We will now test if a binary lens fit may improve this $\chi^{2}$ by more than some threshold level. If this were the case, then a planet signal is probably buried in the small residuals, similarly to the MOA 2007-BLG400 (Dong et al. 2009) and MOA 2008-BLG-310 (Janczak et al. 2010) events.

Our exploration of the parameter space uses the $q w \alpha$ parameterization introduced and justified by Dong et al. (2009). $q$ is the mass ratio between the two components of the lens system $(q<1), w$ is the width of the central caustic as defined by Chung et al. (2005) (given there by their Eq. (12), and called vertical width or short diameter) and $\alpha$ is the trajectory angle with respect to the line joining the two components.

In order to choose the limits of the grid in $\log q$ and $\log w$, we note that any planetary deviation would be strongly smeared out by the extended-source effect when the caustic width $w \ll \rho_{*}$. Han \& Kim (2009) argue that a 5\% deviation can be detected even when the central caustic width $w$ is larger than a quarter of the source diameter $\left(0.5 \rho_{*}\right)$. Indeed, with adequate photometric accuracy, Dong et al. (2009) clearly detect a planetary deviation in MOA 2007-BLG-400 at $w=0.4 \rho_{*}$, and Batista et al. (2009) find some sensitivity even down to $0.1 \rho_{*}$.

We therefore use a grid with $w$ between $10^{-3}$ and $10^{-2}$ and $q$ between $10^{-4}$ and $10^{-2}$. We find a marginal improvement of 55 in $\chi^{2}$ in a region centred around $\log q=-2.5, \log w=-2.5$ and $\alpha=-0.459 \mathrm{rad}$, corresponding to $w=0.14 \rho_{*}$. However, the corresponding residuals do not show any improvement over the single lens case, so we conclude that any possible planetary deviation is not detectable in this event, given the large source size.

A similar conclusion can be drawn even for the presence of multiple planetary companions. Just like in the single-planet case, in a high-magnification event such as ours lens companions would in effect change the point-like caustic of the single lens to a small cusped caustic at the same position. On the light curve this would produce symmetrically placed perturbations at the times of entering and exiting the limb of the source, a region where we see no significant systematic residuals.

\section{Other potential systematics}

In order to affect our limb-darkening measurement and the subsequent $T_{\text {eff }}$ estimate, any potential modelling systematic uncertainty would have to influence the light-curve shape in the narrow region corresponding to the caustic crossing. In our case the region is limited to the $\lesssim 1$-day-wide interval around the peak. Having already tested negatively for the presence of a planetary companion, we turn here to potential uncertainties related to the source, and to the limb-darkening treatment.

Two relevant source-related uncertainties that could influence the result are the presence of star spots and the presence of low-level variability of the source star. The microlensing influence of a star spot would be temporally limited to the spotcrossing time, and the maximum photometric effect is given approximately by the fractional radius of the spot (Heyrovský $\&$ Sasselov 2000). Judging by the amplitude of the residuals in the peak region, our event could potentially permit spots with radii $<0.01$ stellar radii. Nevertheless, any such a spot, in addition to being obscured by light-curve noise, would affect only 0.01 of the source-crossing time. For a longer duration small-amplitude effect one would be limited to the option of a very low-contrast larger spot possibly positioned off the projected lens path. However, such a weak perturbation would be unlikely to affect our results significantly.

Potential low-level variability of the source should always be considered, especially because it could go undetected in the absence of the lens if there is substantial blended light from other stars. In the presence of the lens it should be noticeable in the residuals near the light-curve peak, which should also be mutually correlated in the different light curves as seen in the MACHO Alert 95-30 case (Alcock et al. 1997; Heyrovský 2003). However, only a source varying significantly on timescales $\lessgtr$ the source-crossing time could potentially affect the limb-darkening measurement. Checking our residuals in the peak region, we find that they stay below the $1 \%$ level and the 
residuals from individual curves do not exhibit a clear correlated pattern.

The results could be potentially affected also by the adopted limb-darkening treatment. For our measurement we have chosen the linear limb-darkening law primarily because it is the most widely used single-parameter law. In addition, it is reasonably accurate for broadband photometry in the optical part of the spectrum of a wide range of stars. For the sub-grid of Kurucz models tested in the paper and the relevant photometric bands, the accuracy of the linear law stays better than its average value for the full Kurucz grid (Heyrovský 2007). Within the tested range, the accuracy is best in the $V_{\mathrm{s}}$-band and decreases with effective wavelength to the $I$-band. Nevertheless, our $V_{\mathrm{s}}$-band and $I_{\mathrm{s}}$-band measurements gave us compatible results. Moreover, in order to compensate for the potential inadequacy of the law, we compared the measured limb darkening not only with the LLDCs of the Kurucz models but also directly with their unapproximated limb-darkening profiles. In principle, one could test more advanced limb-darkening laws such as the PCA description (Heyrovský 2003, 2008) or higher-order analytical laws with more free parameters. However, non-negligible inadequacy of the linear law would be exhibited by a centre-to-limb pattern in the residuals, something we do not see in our results given the level of light-curve noise. Based on the above we do not expect the limb-darkening treatment to change our conclusions on the measured limb darkening and the derived effective temperature of the source star.

Finally, it should be noted that inaccuracies may arise on the model-atmosphere side of the comparison. The Kurucz ATLAS9 models used in our analysis involve various simplifications, such as 1-D radiative transfer in a plane-parallel atmosphere, the assumption of local thermodynamic equilibrium, or the mixinglength treatment of convection. Such approximations may affect the predicted limb-darkening profiles, which reflect the change of physical conditions in the atmosphere with depth. Accurate measurement of limb darkening thus provides an opportunity to test the depth structure of model atmospheres and, hence, the appropriateness of their underlying physical assumptions.

\section{Summary and conclusion}

We have performed dense photometric monitoring of the microlensing event OGLE 2008-BLG-290, a short duration and very small impact parameter microlensing event generated by a point-like lens transiting a giant star. The peak magnification was about $A\left(t_{0}\right) \sim 100$, effectively multiplying the diameter of our network telescopes by a factor $\sim 10$. Using a calibrated colourmagnitude diagram analysis and isochrones, we find a source angular radius $\theta_{*} \simeq 6.5 \pm 0.4 \mu \mathrm{as}$, and a physical radius $R_{*} \simeq 10 R_{\odot}$ at the adopted source distance $D_{\mathrm{S}}=7.6 \mathrm{kpc}$. A Galaxy model together with the event time-scale $t_{\mathrm{E}}=16.4$ days and the angular Einstein radius $\theta_{\mathrm{E}}=0.3$ mas yielded statistically inferred median parameters, namely a lens mass $M \sim 0.3 M_{\odot}$, a lens distance $D_{\mathrm{L}} \sim 6.0 \mathrm{kpc}$, and a relative velocity $v_{\perp} \sim 190 \mathrm{~km} \mathrm{~s}^{-1}$.

From our photometric data, we have derived accurate measurements of the linear limb-darkening coefficients of the source for the $V_{\mathrm{s}}$, SDSS $r, R$, MOA-red, $I_{\mathrm{s}}$, and $I$ broadband filters. The obtained limb-darkening profiles lead to a source-star temperature estimate of $T_{\text {eff }}=4700_{-200}^{+100} \mathrm{~K}$ when compared with the limb-darkening profiles of Kurucz's ATLAS9 atmosphere models. This result is in marginal disagreement with the corresponding estimate based on measured colours, a finding that has already been noted for several previous events measured by the microlensing technique, which also parallels the known discrepancies in temperature estimates based on photometry vs. spectroscopy of similar cool giants (Fulbright et al. 2006). Possible explanations involve reddening corrections which may lead to wrong colour estimates, or inaccuracies in model atmosphere physics.

Acknowledgements. We express our gratitude to ESO for a two months invitation at Santiago headquarters, Chile, where part of this work was achieved. We are very grateful to the observatories that support our science (Bronberg, Canopus, CTIO, ESO, IRSF, LCOGT, Liverpool, LOAO, MOA, OGLE, Perth, SAAO, Skinakas) via the generous allocation of time that makes this work possible. The operation of Canopus Observatory is in part supported by a financial contribution from David Warren. The OGLE project is partially supported by the Polish MNiSW grant N20303032/4275 to AU. Allocation of the Holmes grant from the French Agence Nationale de la Recherche has been indispensable to finance observing trips and meeting travels, and is gratefully acknowledged here. D.H. was supported by Czech Science Foundation grant GACR 205/07/0824 and by the Czech Ministry of Education project MSM0021620860. C.H. was supported by the grant 2009-0081561 of National Research Foundation of Korea. T.C.H. was financed for his astronomical research at the Armagh Observatory by the Department for Culture, Arts and Leisure, Northern Ireland, UK. D.R. and J.S. acknowledge support from the Communauté française de Belgique Actions de recherche concertées - Académie universitaire Wallonie-Europe. The Dark Cosmology Centre is funded by the Danish National Research Council. P.F. wishes to thank Noriyuki Matsunaga for discussions about the interplay between adopted distance and derived extinction, David Nataf for measuring the red giant clump in the recently released OGLE-III photometric catalogue, and Etienne Bachelet for checking the whole chain from extinction to source size using a refined method. We are grateful to the anonymous referee for constructive comments that helped us improve the manuscript.

This publication makes use of data products from the 2MASS project, as well as the SIMBAD database, Aladin and Vizier catalogue operation tools (CDS Strasbourg, France). The Two Micron All Sky Survey is a joint project of the University of Massachusetts and the Infrared Processing and Analysis Center/California Institute of Technology, funded by the National Aeronautics and Space Administration and the National Science Foundation.

\section{References}

Abe, F., Bennett, D., Bond, I., et al. 2003, A\&A, 411, L493

Affer, L., Micela, G., Morel, T., Sanz-Forcada, J., \& Favata, F. 2005, A\&A, 433, 647

Afonso, C., Alard, C., Albert, J. N., et al. 2000, ApJ, 532, 340

Albrow, M. D., Beaulieu, J.-P., Caldwell, J. A. R., et al. 1999, ApJ, 522, 1011

Albrow, M. D., Beaulieu, J.-P., Caldwell, J. A. R., et al. 2000, ApJ, 534, 894

Albrow, M. D., An, J., Beaulieu, J.-P., et al. 2001, ApJ, 549, 759

Albrow, M. D., Horne, K., Bramich, D. M., et al. 2009, MNRAS, 397, 2099

Alcock, C., Allen, W. H., Allsman, R. A., et al. 1997, ApJ, 491, 436

An, J. H., Albrow, M. D., Beaulieu, J.-P., et al. 2002, ApJ, 572, 521

Aufdenberg, J. P., Mérand, A., Coudé du Foresto, V., et al. 2006, ApJ, 645, 664

Batista, V., Dong, S., Gould, A., et al. 2009, A\&A, 508, 467

Beaulieu, J.-P., Bennett, D. P., Fouqué, P., et al. 2006, Nature, 439, 437

Bennett, D. P., Bond, I. A., Udalski, A., et al. 2008, ApJ, 684, 663

Bessell, M. S., \& Brett, J. M. 1988, PASP, 100, 1134

Bonatto, C., Bica, E., \& Girardi, L. 2004, A\&A, 415, 571

Bond, I. A., Udalski, A., Jaroszyński, M., et al. 2004, ApJ, 606, L155

Bramich, D. M. 2008, MNRAS, 386, L77

Burns, D., Baldwin, J. E., Boysen, R. C., et al. 1997, MNRAS, 290, L11

Cassan, A., Beaulieu, J. P., Brillant, S., et al. 2004, A\&A, 419, L1

Cassan, A., Beaulieu, J.-P., Fouqué, P., et al. 2006, A\&A, 460, 277

Chung, S.-J., Han, C., Park, B.-G., et al. 2005, ApJ, 630, 535

Claret, A. 2000, A\&A, 363, 1081

Dominik, M. 1998, A\&A, 329, 361

Dominik, M. 2006, MNRAS, 367, 669

Dong, S., Bond, I. A., Gould, A., et al. 2009, ApJ, 698, 1826

Dutra, C. M., Santiago, B. X., Bica, E. L. D., \& Barbuy, B. 2003, MNRAS, 338, 253

Eisenhauer, F., Genzel, R., Alexander, T., et al. 2005, ApJ, 628, 246

Fields, D. L., Albrow, M. D., An, J., et al. 2003, ApJ, 596, 1305

Fulbright, J. P., McWilliam, A., \& Rich, R. M. 2006, ApJ, 636, 821

Gaudi, B. S., Bennett, D. P., Udalski, A., et al. 2008, Science, 319, 927

Gould, A., Udalski, A., An, D., et al. 2006, ApJ, 644, L37

Gould, A., Dong, S., Bennett, D. P., et al. 2010, ApJ, 710, 1800

Groenewegen, M. A. T. 2004, MNRAS, 353, 903

Han, C., \& Kim, D. 2009, ApJ, 693, 1835 
Heyrovský, D. 2003, ApJ, 594, 464

Heyrovský, D. 2007, ApJ, 656, 483

Heyrovský, D. 2008, in Proceedings of the Manchester Microlensing Conference, ed. E. Kerins, S. Mao, N. Rattenbury, \& Ł. Wyrzykowski, Manchester, Univ. of Manchester, Proceedings of Science (GMC8) 028 Heyrovský, D., \& Sasselov, D. 2000, ApJ, 529, 69

Heyrovský, D., Sasselov, D., \& Loeb, A. 2000, ApJ, 543, 406 Houdashelt, M. L., Bell, R. A., \& Sweigart, A. V. 2000, AJ, 119, 1448

Janczak, J., Fukui, A., Dong, S., et al. 2010, ApJ, 711, 731

Jiang, G., DePoy, D. L., Gal-Yam, A., et al. 2004, ApJ, 617, 1307

Kervella, P., \& Fouqué, P. 2008, A\&A, 491, 855

Kubas, D., Cassan, A., Beaulieu, J., et al. 2005, A\&A, 435, 941

Kurucz, R. 1993a, Limbdarkening for $2 \mathrm{~km} \mathrm{~s}^{-1}$ grid (No. 13): [+1.0] to $[-1.0]$, Kurucz CD-ROM No. 16, Cambridge, Mass.: Smithsonian Astrophysical Observatory, 16

Kurucz, R. 1993b, Limbdarkening for $2 \mathrm{~km} \mathrm{~s}^{-1}$ grid (No. 13): [+0.0] to $[-5.0]$, Kurucz CD-ROM No. 17, Cambridge, Mass.: Smithsonian Astrophysical Observatory, 17

Kurucz, R. 1994, Solar abundance model atmospheres for 0, 1, 2, 4, $8 \mathrm{~km} \mathrm{~s}^{-1}$. Kurucz CD-ROM No. 19, Cambridge, Mass.: Smithsonian Astrophysical Observatory, 19

Marigo, P., Girardi, L., Bressan, A., et al. 2008, A\&A, 482, 883

Nishiyama, S., Nagata, T., Kusakabe, N., et al. 2006, ApJ, 638, 839

Nishiyama, S., Nagata, T., Tamura, M., et al. 2008, ApJ, 680, 1174

Paczyński, B. 1986, ApJ, 304, 1

Paczyński, B. 1996, ARA\&A, 34, 419

Paczyński, B., \& Stanek, K. Z. 1998, ApJ, 494, L219

Perrin, G., Ridgway, S. T., Coudé du Foresto, V., et al. 2004, A\&A, 418, 675

Popper, D. M. 1984, AJ, 89, 132

Popper, D. M., \& Etzel, P. B. 1981, AJ, 86, 102

Rattenbury, N. J., Mao, S., Sumi, T., \& Smith, M. C. 2007, MNRAS, 378, 1064

Richichi, A., \& Lisi, F. 1990, A\&A, 230, 355

Schultheis, M., Ganesh, S., Simon, G., et al. 1999, A\&A, 349, L69

Southworth, J., Smalley, B., Maxted, P. F. L., Claret, A., \& Etzel, P. B. 2005, MNRAS, 363, 529

Stanek, K. Z., \& Garnavich, P. M. 1998, ApJ, 503, L131

Sumi, T., Bennett, D. P., Bond, I. A., et al. 2010, ApJ, 710, 1641

Tokunaga, A. T., Simons, D. A., \& Vacca, W. D. 2002, PASP, 114, 180

Udalski, A. 2003, Acta Astron., 53, 291

Udalski, A., Jaroszyński, M., Paczyński, B., et al. 2005, ApJ, 628, L109

Witt, H. J. 1995, ApJ, 449, 42

Wittkowski, M., Aufdenberg, J. P., Driebe, T., et al. 2006, A\&A, 460, 855

Wozniak, P. R. 2000, Acta Astron., 50, 421

Yee, J. C., Udalski, A., Sumi, T., et al. 2009, ApJ, 703, 2082

Yoo, J., DePoy, D. L., Gal-Yam, A., et al. 2004, ApJ, 603, 139

Zub, M., Cassan, A., Heyrovský, D., et al. 2009, A\&A, submitted [arXiv:0912.2312]

1 Probing Lensing Anomalies Network, http://planet.iap. fr

2 Microlensing Follow Up Network, http://wWw.astronomy. ohio-state. edu/ microfun

3 The Optical Gravitational Lensing Experiment, http://ogle. astrouw . edu.pl

4 Microlensing Observations in Astrophysics, http://www.phys. canterbury .ac.nz/moa

5 Robotic Telescope Network, http://robonet. lcogt.net

6 Microlensing Network for the Detection of Small Terrestrial Exoplanets, http://WWW . mindstep-science . org

7 Department of Astronomy, Ohio State University, 140 West 18th Avenue, Columbus, OH 43210, USA

8 University of Canterbury, Department of Physics \& Astronomy, Private Bag 4800, Christchurch 8020, New Zealand

9 European Southern Observatory (ESO), Casilla 19001, Vitacura 19, Santiago, Chile

10 European Southern Observatory (ESO), Karl-Schwarzschild-Straße 2, 85748 Garching bei Mun̈chen, Germany

11 Institut d'Astrophysique de Paris, CNRS, Université Pierre \& Marie Curie, 98bis Bd Arago, 75014 Paris, France

12 Astronomisches Rechen-Institut (ARI), Zentrum für Astronomie der Universität Heidelberg (ZAH), Mönchhofstrasse 12-14, 69120 Heidelberg, Germany

13 Scottish Universities Physics Alliance, School of Physics \& Astronomy, University of St Andrews, North Haugh, St Andrews, KY16 9SS, UK
14 University of Notre Dame, Department of Physics, 225 Nieuwland Science Hall, Notre Dame, IN 46556, USA

15 University of Texas, McDonald Observatory, 16120 St Hwy Spur 78, Fort Davis TX 79734, USA

16 Institute of Geophysics and Planetary Physics (IGPP), L-413, Lawrence Livermore National Laboratory, PO Box 808, Livermore, CA 94551, USA

17 Physics Department, Faculty of Arts and Sciences, University of Rijeka, Omladinska 14, 51000 Rijeka, Croatia

18 Technical University of Vienna, Dept. of Computing, Wiedner Hauptstrasse 10, Vienna, Austria

19 School of Mathematics and Physics, University of Tasmania, Private Bag 37, Hobart, Tasmania 7001, Australia

20 NASA Exoplanet Science Institute, Caltech, MS 100-22, 770 South Wilson Avenue, Pasadena, CA 91125, USA

21 Perth Observatory, Walnut Road, Bickley, Perth 6076, Australia

22 South African Astronomical Observatory, PO Box 9 Observatory 7925, South Africa

23 Space Telescope Science Institute, 3700 San Martin Drive, Baltimore, MD 21218, USA

24 Department of Physics, Institute for Basic Science Research, Chungbuk National University, Chongju 361-763, Korea

25 Korea Astronomy and Space Science Institute, 61-1, Whaam-Dong, Youseong-Gu, Daejeon 305-348, Korea

26 Bronberg Observatory, Pretoria, South Africa

27 Warsaw University Observatory. Al. Ujazdowskie 4, 00-478 Warszawa, Poland

28 Universidad de Concepción, Departamento de Física, Astronomy Group, Casilla 160-C, Concepción, Chile

29 Institute of Astronomy, University of Cambridge, Madingley Road, Cambridge CB3 0HA, UK

30 Institute of Information and Mathematical Sciences, Massey University, Private Bag 102-904, North Shore Mail Centre, Auckland, New Zealand

31 Solar-Terrestrial Environment Laboratory, Nagoya University, Nagoya, 464-8601, Japan

32 Jodrell Bank Centre for Astrophysics, University of Manchester, Manchester M13 9PL, UK

33 Department of Physics, Konan University, Nishiokamoto 8-9-1, Kobe 658-8501, Japan

34 Nagano National College of Technology, Nagano 381-8550, Japan

35 Department of Physics, University of Auckland, Private Bag 92019, Auckland 1142, New Zealand

36 Tokyo Metropolitan College of Industrial Technology, Tokyo 1160003, Japan

37 Department of Physics and Astrophysics, Faculty of Science, Nagoya University, Nagoya 464-8602, Japan

38 Mount John Observatory, PO Box 56, Lake Tekapo 8770, New Zealand

39 Las Cumbres Observatory, 6740B Cortona Dr, suite 102, Goleta, CA 93117, USA

40 Astrophysics Research Institute, Liverpool John Moores University, Twelve Quays House, Egerton Wharf, Birkenhead CH41 1LD, UK

41 School of Physics, University of Exeter, Stocker Road, Exeter EX4 4QL, UK

42 Department of Physics, University of Warwick, Coventry, CV4 7AL, UK

43 Department of Physics, Broida Hall, University of California, Santa Barbara CA 93106-9530, USA

44 LATT, Université de Toulouse, CNRS, 14 avenue Edouard Belin, 31400 Toulouse, France

45 Dark Cosmology Centre, Københavns Universitet, Juliane Maries Vej 30, 2100 København, Denmark

46 University Observatory Munich, Scheinerstrasse 1, 81679 München, Germany

47 Max Planck Institute for Extraterrestrial Physics, Giessenbachstrasse, 85748 Garching, Germany

48 Institute of Theoretical Physics, Charles University, V Holešovičkách 2, 18000 Prague, Czech Republic 
49 Bellatrix Observatory, via Madonna de Loco 47, 03023 Ceccano, Italy

50 Dipartimento di Fisica, Universita' di Salerno and INFN, sez. di Napoli, Italy

51 Armagh Observatory, College Hill, Armagh, BT61 9DG, Ireland

52 Department of Physics, Sharif University of Technology, PO Box 11155-9161, Tehran, Iran

53 Institut für Astrophysik, Georg-August Universität, Friedrich-HundPlatz 1, 37077 Göttingen, Germany

54 Institut d'Astrophysique et de Géophysique, Allée du 6 Août, Sart Tilman, Bât. B5c, 4000 Liège, Belgium

55 Astrophysics Group, Keele University, Newcastle-under-Lyme, ST5 5BG, UK
56 INAF, Osservatorio Astronomico di Brera, via E. Bianchi 46, 23807 Merate (LC), Italy

57 Deutsches SOFIA Institut, Universität Stuttgart, Pfaffenwaldring 31, 70569 Stuttgart, Germany

58 SOFIA Science Center, NASA Ames Research Center, Mail Stop N211-3, Moffett Field CA 94035, USA

59 Department of Astronomy, Kyoto University, Kyoto 606-8502, Japan

${ }^{60}$ School of Mathematical Sciences, Queen Mary University of London, Mile End Road, London E1 4NS, UK

61 The Wendelstein Calar Alto Pixellensing Project, http://www. usm. uni-muenchen.de/people/arri/wecapp.html 